\title{
UV-photoprocessing of interstellar ice analogs: Detection of hexamethylenetetramine-based species
}

\author{
G. M. Muñoz Caro ${ }^{1,3}$, U. Meierhenrich ${ }^{2}$, W. A. Schutte ${ }^{1}$, W. H.-P. Thiemann ${ }^{2}$, and J. M. Greenberg ${ }^{1, \star}$ \\ 1 Raymond and Beverly Sackler Laboratory for Astrophysics, Leiden Observatory, 2300 RA Leiden, The Netherlands \\ 2 Universität Bremen, NWII, Dept. Phys. Chemistry, Leobener Straße, 28359 Bremen, Germany \\ 3 Institut d'Astrophysique Spatiale, UMR 8617, Bât. 121, Campus Paris XI, 91405 Orsay, France
}

Received 15 November 2002 / Accepted 12 August 2003

\begin{abstract}
The physical conditions governing the dense cloud environment are reproduced in a high vacuum experimental setup at low temperature $T \approx 12 \mathrm{~K}$. The accretion and photoprocessing of ices on grain surfaces is simulated by depositing an ice layer on a cold finger, while it is irradiated by ultraviolet (UV) photons. After irradiation the sample is slowly warmed to room temperature; a residue remains, containing the most refractory products of photo- and thermal processing. In this paper we report on the analysis of the residues performed by means of gas chromatography-mass spectrometry (GC-MS). A number of new molecules based on hexamethylenetetramine (HMT, $\mathrm{C}_{6} \mathrm{H}_{12} \mathrm{~N}_{4}$ ), the most abundant component of the residues reported here, were detected: methyl-HMT $\left(\mathrm{C}_{6} \mathrm{H}_{11} \mathrm{~N}_{4}-\mathrm{CH}_{3}\right)$, hydroxy-HMT $\left(\mathrm{C}_{6} \mathrm{H}_{11} \mathrm{~N}_{4}-\mathrm{OH}\right)$, methanyl-HMT $\left(\mathrm{C}_{6} \mathrm{H}_{11} \mathrm{~N}_{4}-\mathrm{CH}_{2} \mathrm{OH}\right)$, amin-aldehyd-HMT $\left(\mathrm{C}_{6} \mathrm{H}_{11} \mathrm{~N}_{4}-\mathrm{NH}-\mathrm{CHO}\right)$ and methanyl-aldehyd-HMT $\left(\mathrm{C}_{6} \mathrm{H}_{11} \mathrm{~N}_{4}-\mathrm{CHOH}-\mathrm{CHO}\right)$. To the best of our knowledge, this is the first reported synthesis of these molecules. Currently, these are the heaviest identified components of the residue. These species might also be present in the interstellar medium, given that the ice was submitted to high temperatures, of the order of $300 \mathrm{~K}$, and form part of comets. Our work serves as preparation for the ESA-Rosetta mission, which plans to do in situ analysis of the composition of a comet nucleus with the COSAC instrumentation.
\end{abstract}

Key words. ISM: molecules - methods: laboratory - ultraviolet: ISM - ISM: dust, extinction

\section{Introduction}

Dense molecular clouds in the interstellar medium (ISM), with densities ranging from $10^{3}-10^{6}$ molecules $\mathrm{cm}^{-3}$ and kinetic temperatures as low as $10 \mathrm{~K}$, are the sites of star formation. Gas phase molecules accrete onto dust particles forming ice mantles with $\mathrm{H}_{2} \mathrm{O}, \mathrm{CO}, \mathrm{CO}_{2}, \mathrm{CH}_{3} \mathrm{OH}$, and $\mathrm{NH}_{3}$ as the main molecular components (Gibb et al. 2000, 2001). Ice-coated dust particles in dense clouds can be exposed to UV photons and cosmic rays (Muñoz Caro et al. 2001; Mennella et al. 2001), leading to the formation of new molecules, radicals, and other fragments. A compilation of the environments within dense clouds in which energetic processing can be relevant is given elsewhere (Muñoz Caro \& Schutte 2003).

Laboratory experiments simulating the energetic processing of interstellar ice analogs show that, as a result, organic species are formed with high molecular mass, up to about 400 amu (Greenberg \& Yencha 1973; Hagen et al. 1979; Agarwal et al. 1985; d'Hendecourt et al. 1986; Allamandola et al. 1988; Briggs et al. 1992; Bernstein et al. 1995; Cottin et al. 2001; Bernstein et al. 2002; Muñoz Caro et al. 2002).

Send offprint requests to: G. M. Muñoz Caro,

e-mail: Guillermo.Munoz-Caro@ias.u-psud.fr

$\star$ Deceased.
Hexamethylenetetramine $\left[\left(\mathrm{CH}_{2}\right)_{6} \mathrm{~N}_{4}, \mathrm{HMT}\right]$ was first detected in the residues by Briggs et al. (1992). It is the main refractory product of photolysis of ice mixtures containing $\mathrm{CH}_{3} \mathrm{OH}$ (Bernstein et al. 1995; Muñoz Caro \& Schutte 2003). These results indicate that interstellar ices could be the birthplaces of complex organic molecules. This possibly has important implications for the composition of the dust in the ISM and in the solar nebula, and even for the origin of life (Oró 1961; Greenberg 1986; Bernstein et al. 2002; Muñoz Caro et al. 2002).

Comets are thought to be formed by aggregation of dust particles in the solar nebula, and probably host the most pristine material in the solar system. The large abundances of oxygenrich complex organic molecules found in comet Halley (Kissel \& Krueger 1987; Fomenkova et al. 1994) suggest that energetic processing in the ISM, and/or the solar nebula, cannot be disregarded.

This article reports on the experimental simulation of ice photoprocessing in circumstellar/interstellar regions. The remaining products at room temperature, henceforth called residue, were analyzed by gas chromatography-mass spectroscopy (GC-MS). Five new species related to HMT were detected in the residues. The aim of these experiments was to study the kind of organic species that could be detected in comets by in situ analysis, as planned by the COSAC 
(Cometary Sampling and Composition Experiment) team of the Rosetta mission (Rosenbauer et al. 2003). For this reason we have limited ourselves either to plain GC-MS, or to GC-MS after derivatization with dimethylformamide dimethylacetal (DMF-DMA), the reagent that will be employed by COSAC (Meierhenrich et al. 2001).

An ice mixture of molar composition $\mathrm{H}_{2} \mathrm{O}: \mathrm{CH}_{3} \mathrm{OH}: \mathrm{NH}_{3}: \mathrm{CO}: \mathrm{CO}_{2}=2: 1: 1: 1: 1$ was selected as standard. $\mathrm{H}_{2} \mathrm{O}: \mathrm{CH}_{3} \mathrm{OH}: \mathrm{CO}_{2}=2: 1: 1$ ice resembles the ice composition found close to protostellar sources (Gerakines et al. 1999; Ehrenfreund et al. 1999; Dartois et al. 1999; Gibb et al. 2001). It should be noted that the line of sight abundances of $\mathrm{CH}_{3} \mathrm{OH}$ and $\mathrm{CO}_{2}$ are $~ 5-10$ times lower due to the dominance of $\mathrm{H}_{2} \mathrm{O}$ ice in the cold outer regions (Gerakines et al. 1999). Recent results show that, contrary to earlier views (Gibb et al. 2001), most of the ammonia towards embedded YSOs may have been converted to ammonium $\left(\mathrm{NH}_{4}^{+}\right)$by acid-base reactions following the initial accretion (Schutte \& Khanna 2003; Taban et al. 2003).

The layout of this paper is as follows: in Sect. 2 we describe the experimental protocol and the GC-MS analysis. The experimental results are presented and discussed in Sect. 3. The astrophysical implications are derived in Sect. 4.

\section{Experimental}

\subsection{Experimental protocol}

The experimental setup is made of stainless steel. It consists of a high vacuum chamber where a gas mixture is deposited on a cold finger and irradiated. The system is pumped by a turbo pump (Pfeiffer Balzers TSH $280 \mathrm{H}$ ) backed up by a diaphragm pump (Vacuubran MD4T) in order to mimic the interstellar vacuum. The pressure of the system at room temperature is $P \approx 1 \times 10^{-7}$ torr. The low temperatures typical of dense clouds, ranging from 10 to $50 \mathrm{~K}$, are achieved by means of a closed-cycle helium cryostat (Air Products Displex DE-202). At the cold finger the temperature is $T \approx 12 \mathrm{~K}$. For a detailed description, see Gerakines et al. (1995).

The gas mixture is prepared by filling a bulb with different gases of increasing vapor pressures. The vacuum pressure of the gas line is $P \approx 10^{-5}$ torr. The gas mixtures prepared for our experiments contained, in various proportions, $\mathrm{H}_{2} \mathrm{O}$ (liquid), distilled; $\mathrm{CH}_{3} \mathrm{OH}$ (liquid), Janssen Chimica 99.9\%; $\mathrm{NH}_{3}$ (gas), Praxair 99.999\%; CO (gas), Praxair 99.997\%; and $\mathrm{CO}_{2}$ (gas), Praxair $99.996 \% . \mathrm{CO}_{2}$ was kept in a separate bulb in order to prevent it from reacting with the ammonia, $\mathrm{NH}_{3}$, with deposition through two independent deposition tubes (Gerakines et al. 1995). ${ }^{13} \mathrm{C}$-labelled gases $\left({ }^{13} \mathrm{CO}\right.$ (gas), Cambridge Isotope Laboratories Inc. (C.I.L.) $99 \%{ }^{13} \mathrm{C} ;{ }^{13} \mathrm{CO}_{2}$ (gas), C.I.L. $99 \%{ }^{13} \mathrm{C}$; and ${ }^{13} \mathrm{CH}_{3} \mathrm{OH}$ (liquid), Sigma $99 \%{ }^{13} \mathrm{C}$ ) and ${ }^{15} \mathrm{NH}_{3}$ (gas, C.I.L. $>98 \%{ }^{15} \mathrm{~N}$ ) were used to deduce the number of $\mathrm{C}$ and $\mathrm{N}$ atoms in the products and to search for possible contaminants. Simultaneous to deposition, the ice layer is UV-irradiated with a microwave stimulated hydrogen flow discharge lamp (output $\approx 1.5 \times 10^{15}$ photons $\mathrm{s}^{-1}$, Weber \& Greenberg (1985); $\left.E_{\text {photon }}=7.3-10.5 \mathrm{eV}\right)$.

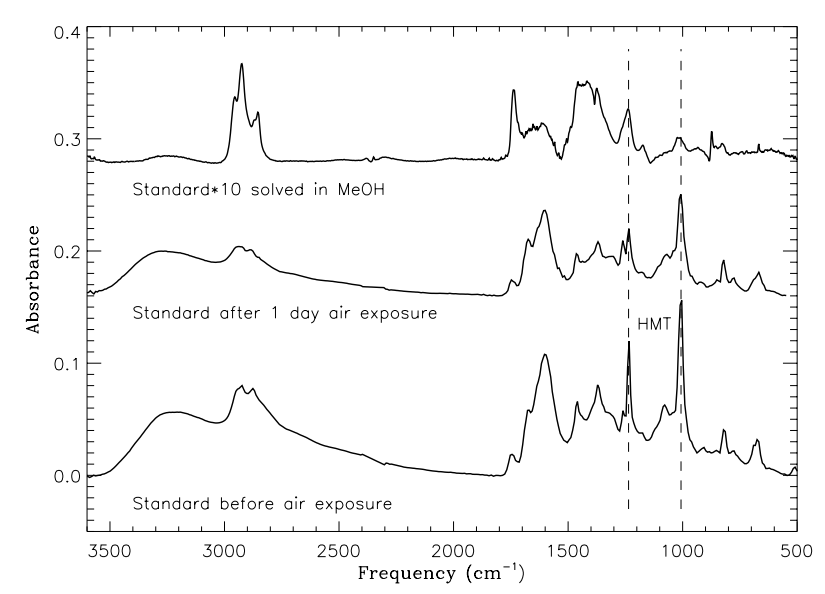

Fig. 1. Residue spectrum, obtained from irradiation of the standard ice mixture, before and after 1 day exposure to air.

The irradiation/deposition time was typically $12 \mathrm{~h}$. The gas flow was $\approx 10^{16}$ molecules $\mathrm{s}^{-1}$. The corresponding average $\mathrm{UV}$ dose is $\approx 0.15 \mathrm{UV}$ photon molecule ${ }^{-1}$. A long simultaneous deposition and irradiation is required to obtain ice samples of sufficient size to yield enough residue for a good analysis. After irradiation the system is warmed up gradually by means of a temperature controller (Scientific Instruments Inc. 9600-1) at $1 \mathrm{~K} \mathrm{~min}^{-1}$ up to $T=40 \mathrm{~K}$, in order to prevent explosive reactions caused by $\mathrm{UV}$-produced free radicals embedded in the ice (d'Hendecourt et al. 1982). Subsequently, the warmup proceeds at about $4 \mathrm{~K} \mathrm{~min}^{-1}$ up to room temperature; at that point the refrigerator and temperature controller are turned off and the system slowly gets in thermal equilibrium with the environment.

\subsection{GC-MS analysis}

The gas molecules condense on an aluminum block mounted on the cold finger at $12 \mathrm{~K}$ while being irradiated. This substrate has 21 circular wells on each face of $4 \mathrm{~mm}$ diameter and $1 \mathrm{~mm}$ depth; before the experiment, it is cleaned using an ultrasonic bath with water and detergent, and rinsed with ethanol, with each cleaning step repeated three times. After the experiment the substrate with the residue is removed from the system and stored inside a capsule in a nitrogen atmosphere, where it is kept for analysis. During the removal, and also just before the analysis, the sample is briefly exposed to air. No effects due to air exposure were observed in the infrared spectra of the residues other than a slight overall decrease of the peak intensities, even for long exposure times of the order of one day (Fig. 1). The relative peak intensities in the infrared vary after solving the sample in methanol, due to the difference in solubility of the spectral components (Fig. 1). This certainly affects the relative abundances of the residue components as measured by GC-MS involving methanol as solvent. The lack of chemical modification by air is consistent with the overall composition of the residue, which is dominated by saturated compounds with carboxylic and amino groups, which do not react with $\mathrm{O}_{2}$ and $\mathrm{N}_{2}$ (Jenniskens et al. 1993). 
Table 1. Log of experiments for GC-MS analysis.

\begin{tabular}{|c|c|c|c|c|c|}
\hline Experiment & $\begin{array}{c}\text { Ice } \\
\mathrm{H}_{2} \mathrm{O}: \mathrm{NH}_{3}: \mathrm{CH}_{3} \mathrm{OH}: \mathrm{CO}: \mathrm{CO}_{2}\end{array}$ & $\begin{array}{c}\text { UV dose } \\
\text { photon molec. }^{-1}\end{array}$ & $\begin{array}{l}\text { Molecular ion mass } \\
\text { amu }\end{array}$ & Assigned carrier & $\begin{array}{c}\text { Integ. area } \\
\text { (relat. to HMT) }\end{array}$ \\
\hline \multirow[t]{4}{*}{1} & $2: 1: 1: 1: 1$ & 0.15 & 140 & HMT & 100 \\
\hline & & & 154 & $\mathrm{HMT}-\mathrm{CH}_{3}$ & 0.005 \\
\hline & & & 156 & HMT-OH & $0.04,0.02$ \\
\hline & & & 198 & HMT-CHOH-CHO & 0.04 \\
\hline \multirow[t]{2}{*}{2} & $2: 1: 1: 1: 1^{2}$ & 0.45 & 144 & HMT & 100 \\
\hline & & & 160 & $\mathrm{HMT}-\mathrm{OH}$ & 2.0 \\
\hline \multirow[t]{2}{*}{3} & $2: 1: 1: 1: 1^{3}$ & 0.28 & $140,141,142,143,144$ & HMT & 100 \\
\hline & & & $156,157,158,159$ & HMT-OH & 4.0 \\
\hline \multirow[t]{5}{*}{4} & $2: 1: 1: 0: 1$ & 0.15 & 140 & HMT & 100 \\
\hline & & & 154 & $\mathrm{HMT}-\mathrm{CH}_{3}$ & 0.07 \\
\hline & & & 156 & HMT-OH & 0.25 \\
\hline & & & 170 & $\mathrm{HMT}-\mathrm{CH}_{2} \mathrm{OH}$ & 0.03 \\
\hline & & & 183 & HMT-NH-CHO & 0.04 \\
\hline \multirow[t]{5}{*}{5} & $2: 1: 1: 0: 1^{4}$ & 0.15 & 146 & HMT & 100 \\
\hline & & & 161 & $\mathrm{HMT}-\mathrm{CH}_{3}$ & $0.02^{1}$ \\
\hline & & & 162 & HMT-OH & 1.2 \\
\hline & & & 177 & $\mathrm{HMT}-\mathrm{CH}_{2} \mathrm{OH}$ & 0.3 \\
\hline & & & 190 & HMT-NH-CHO & 0.1 \\
\hline \multirow[t]{4}{*}{6} & $2: 0.2: 1: 0: 1$ & 0.28 & 140 & HMT & 100 \\
\hline & & & 154 & $\mathrm{HMT}-\mathrm{CH}_{3}$ & 0.01 \\
\hline & & & 156 & HMT-OH & 0.20 \\
\hline & & & 198 & HMT-CHOH-CHO & 0.07 \\
\hline \multirow[t]{4}{*}{7} & $2: 1: 1: 1: 1^{4,5}$ & 0.45 & 146 & HMT & 100 \\
\hline & & & 161 & $\mathrm{HMT}-\mathrm{CH}_{3}$ & 0.4 \\
\hline & & & 162 & HMT-OH & 12 \\
\hline & & & 206 & HMT-CHOH-CHO & 2.0 \\
\hline $\begin{array}{l}\text { Using hexane } \\
{ }^{15} \mathrm{NH}_{3} \text {-labelle } \\
\text { With }{ }^{13} \mathrm{CO},{ }^{13} \\
{ }^{13} \mathrm{C} \text {-labelled. } \\
\text { Using DMF-I }\end{array}$ & $\begin{array}{l}\text { as solvent, undetected in met } \\
\mathrm{CO}_{2},{ }^{12} \mathrm{CH}_{3} \mathrm{OH} \text {. } \\
\text { MA derivatization. }\end{array}$ & solution. & & & \\
\hline
\end{tabular}

Using a pipet, $15 \mu \mathrm{l}$ of solvent were deposited onto one of the wells. One $\mu \mathrm{l}$ of this solution was manually injected into the gas chromatograph (Varian 3400) coupled to a mass spectrometer (Finnigan MAT ITS 40) located at the Dept. of Physical Chemistry in Bremen University. The injection was performed at $200{ }^{\circ} \mathrm{C}$ with helium 5.0 as carrier gas. The oven temperature increased gradually at $2{ }^{\circ} \mathrm{C} \mathrm{min}^{-1}$ from $60{ }^{\circ} \mathrm{C}$ to $170{ }^{\circ} \mathrm{C}$. The mass spectrometer was operated in electron impact (EI) mode applying a voltage of $70 \mathrm{eV}$. The capillary column employed is a Chirasil-Dex CB $(10 \mathrm{~m} \times 0.25 \mathrm{~mm} \times$ $0.25 \mu \mathrm{m}$, Varian-Chrompack). Methanol was used as a polar solvent and $n$-hexane as apolar. The solvents and reagent were purchased from Fluka. The COSAC instrumentation on board Rosetta is designed to identify organic molecules in a comet nucleus by in situ pyrolysis GC-MS (Meierhenrich et al. 2001), assisted by a methylation reagent, dimethylformamide dimethyl-acetal (DMF-DMA, 7.5 molar), which produces methyl esters of polar monomers to increase their volatility. This reagent was tested in Exp. 7 (Table 1).

Analysis of washings of one side of the Al-block where no deposition/irradiation took place were systematically performed under the same analytic conditions. No species were detected in these runs.

\section{Experimental results}

\subsection{Overview of experiments}

Table 1 gives the log of experiments. The first three columns give the experimental parameters, i.e., the experiment number, the starting ice composition and the UV dose. The next three columns give the results, i.e., the mass of the eluting molecule, 
Table 2. Peak identification of the gas chromatogram (corresponding to Exp. 4, Table 1) shown in Fig. 2.

\begin{tabular}{ccccc}
\hline \hline Peak label & $\begin{array}{c}\text { Integ. area } \\
\text { (relat. to HMT) }\end{array}$ & $\begin{array}{c}\text { Separation time } \\
\text { s }\end{array}$ & $\begin{array}{c}\text { Molecular ion mass } \\
\text { amu }\end{array}$ & Assigned carrier \\
\hline 1 & 100 & 1020 & 140 & HMT \\
2 & 0.07 & 1078 & 154 & HMT-CH \\
3 & 0.25 & 1286 & 156 & HMT-OH \\
4 & 0.03 & 1485 & 170 & HMT-CH ${ }_{2}$ OH \\
5 & 0.04 & 1690 & 183 & HMT-NH-CHO \\
Exp. 1 & 0.04 & 3729 & 198 & CH $_{2}$ OH-HMT-CHO/HMT-CHOH-CHO \\
\hline
\end{tabular}

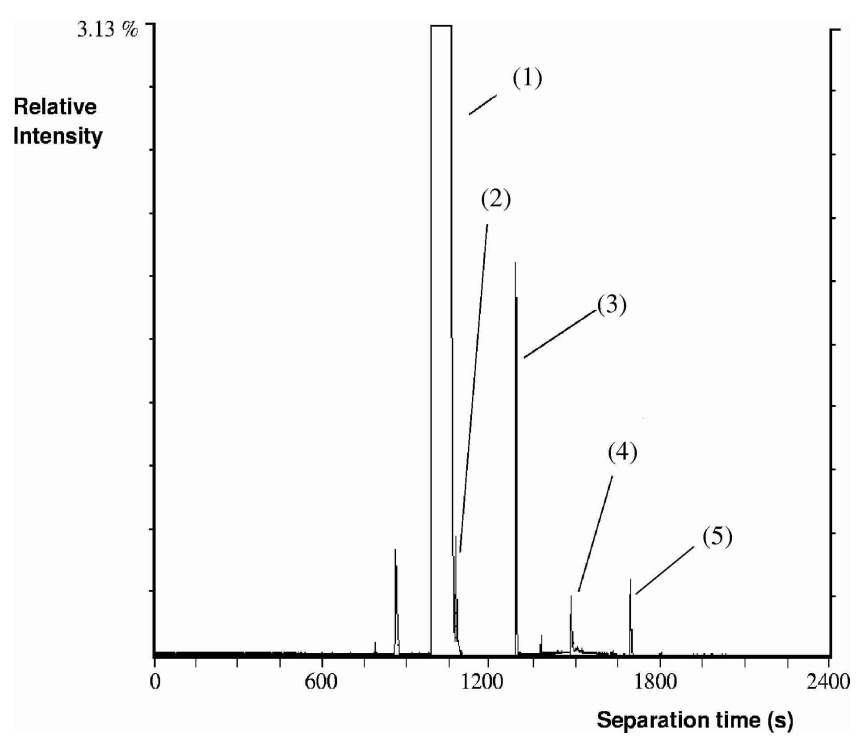

Fig. 2. Gas chromatogram corresponding to the residue obtained from irradiation of the $\mathrm{H}_{2} \mathrm{O}: \mathrm{NH}_{3}: \mathrm{CH}_{3} \mathrm{OH}: \mathrm{CO}=2: 1: 1: 1$ ice mixture. The peak identifications are given in Table 2. Peak 1, identified with HMT, has a relative intensity of $100 \%$ and is shown truncated.

the assigned carrier as deduced from its mass spectrum, and the integrated area relative to HMT. Unless otherwise noted, methanol was used to dissolve the residue for the GC-MS analysis. Experiment 1 is the irradiation of the standard ice mixture. Experiment 2 is the standard labelled with ${ }^{15} \mathrm{~N}$ in order to observe the number of $\mathrm{N}$ atoms contained in the products and to detect possible $\mathrm{N}$-containing contaminants. Experiment 3 contained ${ }^{13} \mathrm{C}$-labelled $\mathrm{CO}$ and $\mathrm{CO}_{2}$, while $\mathrm{CH}_{3} \mathrm{OH}$ was unlabelled; it aimed to reveal the role played by $\mathrm{CH}_{3} \mathrm{OH}$, relative to $\mathrm{CO}$ and $\mathrm{CO}_{2}$, in the formation of the various products. For Exp. 4 the $\mathrm{H}_{2} \mathrm{O}: \mathrm{NH}_{3}: \mathrm{CH}_{3} \mathrm{OH}: \mathrm{CO}_{2}=2: 1: 1: 1$ ice mixture was irradiated; Exp. 5 is the repetition of Exp. 4 with ${ }^{13} \mathrm{C}$-labelled ice, in order to detect possible organic contaminants and to observe the number of $\mathrm{C}$ atoms present in the molecules. Experiment 6 aimed to study the effects of a lower $\mathrm{NH}_{3}$ abundance ( 5 times lower than the standard). Experiment 7 is the ${ }^{13}$ C-labelled standard analyzed after DMF-DMA derivatization, which served as a test of the Rosetta analysis procedure.

The signal-to-noise ratio $(S / N)$ was typically $10^{4}$ for HMT and higher than 10 for the HMT-based molecules, when methanol was used as solvent. On the other hand, the runs performed using hexane were very noisy $(S / N \sim 1$ for the HMT-based molecules).

\subsection{Identification of HMT-based species}

The residues dissolve much better in methanol than in hexane, indicating that they are rather polar. The solution obtained as described in Sect. 2.2 was clearly colored yellow. Water proved to be even a better solvent than methanol, but it is not as suitable for gas chromatography.

Table 1 summarizes the results of the GC-MS analysis. Figure 2 shows the gas chromatogram of the residue obtained from photolysis of the $\mathrm{H}_{2} \mathrm{O}: \mathrm{NH}_{3}: \mathrm{CH}_{3} \mathrm{OH}: \mathrm{CO}=2: 1: 1: 1$ ice mixture (Exp. 4). The identification of the peaks is given in Table 2 and explained below. Clearly, peak 1 dominates, indicating that this compound forms more than $99 \%$ of the residue composition as detected by plain GC-MS analysis, under the described conditions, without derivatization. It is ascribed to HMT, with 140 amu molecular mass. The identification was done by comparison to an external run, using standard HMT (Fluka $>99.5 \%$ ), performed under equal conditions. The shape and retention time, $1020 \mathrm{~s}$, of the compared peaks were identical, as well as the mass spectrum of the carrier molecule. The same identification was made earlier (Briggs et al. 1992; Bernstein et al. 1995). Isotopic labelling using ${ }^{13} \mathrm{C}$ and ${ }^{15} \mathrm{~N}$ of the ice components before irradiation (Exps. 2, 3, and 5) shows the same peak with mass $146 \mathrm{amu}$ and $144 \mathrm{amu}$, respectively, as expected for HMT.

An unidentified peak eluting at $860 \mathrm{~s}$ has a mass of $93 \mathrm{amu}$; it was also found in Exp. 5, ${ }^{13} \mathrm{C}$-labelled, with mass $96 \mathrm{amu}$.

At the tail of the HMT signal appears a small but distinctive peak with an integrated area of $0.07 \%$ relative to HMT, and retention time $1078 \mathrm{~s}$ (Fig. 2, peak 2). The corresponding mass spectrum (Fig. 3, top right panel) shows the parent mass peak at $154 \mathrm{amu}$. The large $140 \mathrm{amu}$ mass fragment is due to the overlap in the retention time of this peak with the HMT peak. This can be seen by taking mass spectra of the peak at different retention times: as the overlap decreases, the 154 amu mass fragment becomes larger relative to the 140 mass peak. A peak at the same retention time was found on the ${ }^{13} \mathrm{C}$-labelled sample with mass 161 amu (Fig. 5, top left panel), showing that the molecule contains seven $\mathrm{C}$ atoms. Unlike for the other detected products, the 154 amu elutant appears to dissolve better in hexane than in methanol (the former gives a higher $S / N$ in the chromatogram), meaning that this component is less polar than the others. Also a better separation of the two chromatographic peaks is obtained using hexane as solvent. For this run, the mass spectrum (not shown) corresponding to the $154 \mathrm{amu}$ molecular ion peak has a 139 amu fragment which is almost as large as the $140 \mathrm{amu}$ mass peak due to HMT, and the $154 \mathrm{amu}$ 

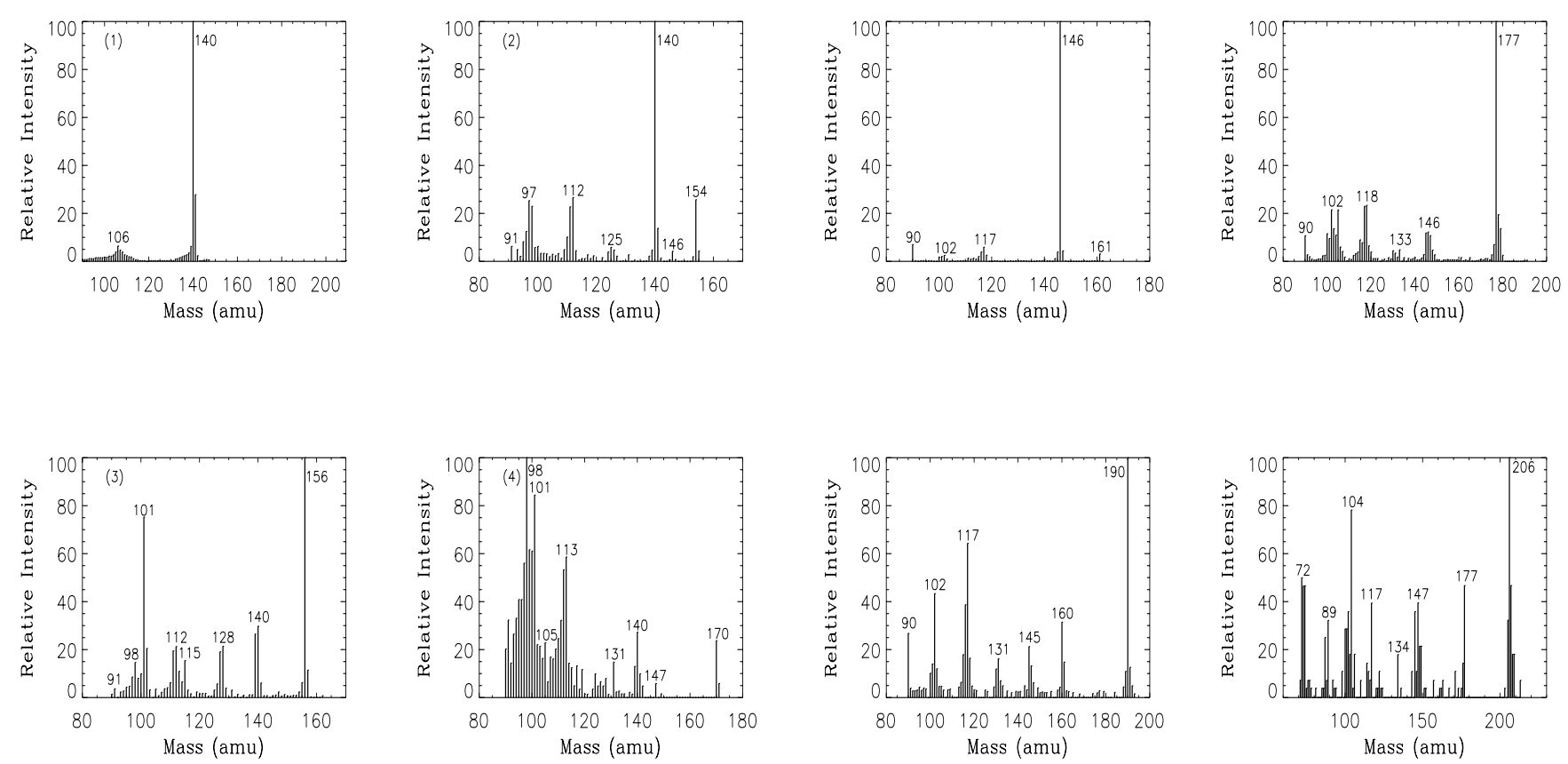

Fig. 5. Mass spectra corresponding to the ${ }^{13} \mathrm{C}$-labelled samples assigned to $\mathrm{CH}_{3}-\mathrm{HMT}$ (top left), $\mathrm{CH}_{2} \mathrm{OH}-\mathrm{HMT}$ (top right), HMT-NH-CHO (bottom left) and HMT-CH(OH)-CHO (bottom
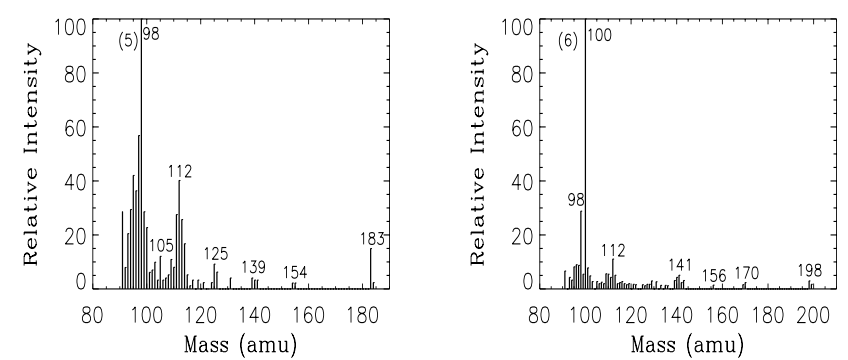

Fig. 3. Mass spectra of detected components assigned to 1. HMT (top left), 2. methyl-HMT (top right), 3. hydroxy-HMT (middle left), 4. methanyl-HMT (middle right), 5. HMT-NH-CHO (bottom left) and 6. HMT- $\mathrm{CH}(\mathrm{OH})-\mathrm{CHO}$ (bottom right).

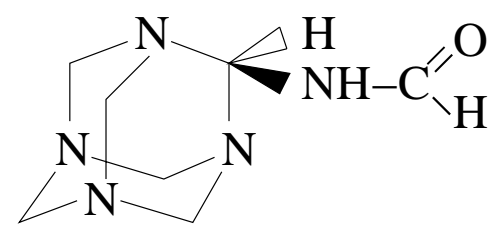

Fig. 4. Representation of amin-aldehyd-hexamethylenetetramine $\left(\mathrm{C}_{6} \mathrm{H}_{11} \mathrm{~N}_{4}-\mathrm{NH}-\mathrm{CHO}\right)$; mass $183 \mathrm{amu}$. Each vertex in the HMT structure corresponds to a $\mathrm{CH}_{2}$ group.

mass peak itself is larger than the $140 \mathrm{amu}$ mass peak. The 139 amu mass fraction (145 amu in the ${ }^{13} \mathrm{C}$-sample) is most probably due to the HMT radical, while the additional $\mathrm{C}$ is in a side group of $15 \mathrm{amu}$. Therefore, the peak is assigned to the substitution of a $\mathrm{H}$ atom by a methyl group $\left(-\mathrm{CH}_{3}\right)$ on the molecular structure of HMT.

Another component elutes at $1286 \mathrm{~s}$, with an integrated area of $0.25 \%$ relative to HMT (Fig. 2, peak 3). The mass spectrum gives a molecular ion peak at 156 amu (Fig. 3, middle left panel). The 139 amu mass fraction, ascribed to HMT right)
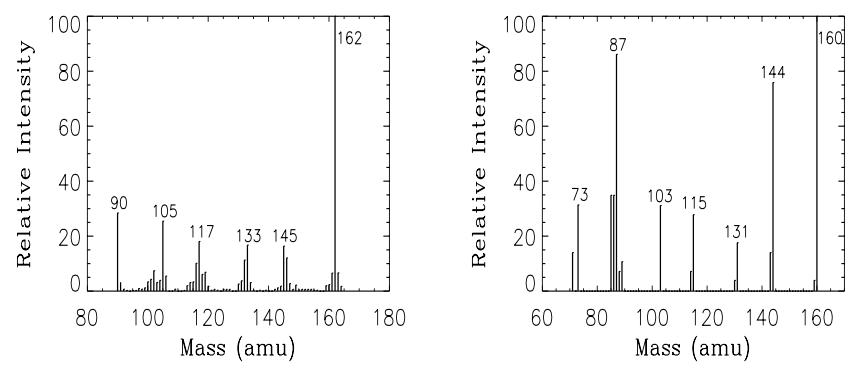

Fig. 6. Mass spectra corresponding to the ${ }^{13} \mathrm{C}$ (left panel) and ${ }^{15} \mathrm{~N}$ (right panel) labelled peaks attributed to HMT-OH.

radical, is also present. For the sample prepared from ${ }^{13} \mathrm{C}$-educts the same gas chromatographic retention was observed, and for the ${ }^{15} \mathrm{~N}$-labelled sample as well. The corresponding mass spectra are shown in Fig. 6. For the ${ }^{13} \mathrm{C}$ sample the shift in mass is due to the six C atoms of HMT. Similarly, the ${ }^{15} \mathrm{~N}$-sample has a mass shift of $4 \mathrm{amu}$ relative to the ${ }^{14} \mathrm{~N}$-sample due to the four $\mathrm{N}$ atoms of HMT. This means that none of these two elements is contained in the side group, and the only alternative left is that a hydroxy $(-\mathrm{OH})$ group replaced a $\mathrm{H}$ atom on the HMT molecule leading to hydroxyHMT (HMT-OH, $156 \mathrm{amu})$.

At retention time $1485 \mathrm{~s}$, a new feature with integrated area $0.03 \%$ relative to HMT appears in the chromatogram (Fig. 2, peak 4). The mass spectrum of this elutant, with molecular ion peak at $170 \mathrm{amu}$ and the presence of the $139 \mathrm{amu}$ fragment, is evidence for the similarity with the HMT structure (Fig. 3, middle right panel). This component is also found at the same retention time for the ${ }^{13} \mathrm{C}$-labelled sample with mass 177 amu (Fig. 5, top right panel), meaning that the side group(s) contains(s) one carbon atom. The constraints could be satisfied by addition of separate methyl $\left(-\mathrm{CH}_{3}\right)$ and 
hydroxy $(-\mathrm{OH})$ groups. However, fragmentation of the molecule would show mass ions at $155\left(\mathrm{M}-\mathrm{CH}_{3}\right), 153(\mathrm{M}-\mathrm{OH})$ and $138\left(\mathrm{M}-\mathrm{CH}_{2}-\mathrm{OH}\right)$. The fact that the first fragmentation step gives mass 139 (mass 140 is probably due to $\mathrm{H}$ addition in the mass spectrometer and 147 is a spurious result which, for instance, does not appear in the mass spectrum of this molecule in the ${ }^{13} \mathrm{C}$-labelled sample) leads to the conclusion that a single $\mathrm{H}$ was substituted in the original HMT molecule by a radical with mass $31 \mathrm{amu}$, which in addition contains one $\mathrm{C}$ atom. This is consistent with methanyl-HMT (HMT- $\left.\mathrm{CH}_{2} \mathrm{OH}\right)$.

The last component elutes at $1690 \mathrm{~s}$ with integrated area $0.04 \%$ relative to HMT (Fig. 2, peak 5). The mass spectrum (Fig. 3, bottom left panel) shows the molecular ion peak at $183 \mathrm{amu}$. Mass $139 \mathrm{amu}$ is due to the HMT radical pointing to one side group attached to the HMT molecule. For the ${ }^{13} \mathrm{C}$-labelled sample there is a peak at the same position with mass 190 amu (Fig. 5, bottom left panel). The mass difference of 7 between the isotopic and non-isotopic samples indicates that there is one $\mathrm{C}$ atom on the radical. To account for a mass of $44 \mathrm{amu}$ this radical should contain a $\mathrm{C}$, a N, and an $\mathrm{O}$ atom. It must therefore be due to HMT-NH-CHO.

For a different run, Exp. 1, corresponding to the standard ice mixture, a molecule with mass 198 amu eluting at $3729 \mathrm{~s}$ with integrated area $0.04 \%$ was detected. This compound was also found on the ${ }^{13} \mathrm{C}$-labelled sample with mass 206 amu at the same retention time. The corresponding mass spectra are given in Figs. 3 and 5. Once more, the 139 amu ion peak assigned to the HMT radical is present and the attached side group(s) contain(s) $2 \mathrm{C}$ atoms. The $170 \mathrm{amu}$ mass is due to addition of a $-\mathrm{CH}_{2} \mathrm{OH}$ radical. The molecular mass of $198 \mathrm{amu}$ is obtained by adding the $-\mathrm{CHO}$ radical of aldehydes. The radicals could be attached to two different carbons of the HMT structure, or form the single radical - $\mathrm{CHOH}-\mathrm{CHO}$. In the latter case breaking of the - $\mathrm{CHO}$ group gives $169 \mathrm{amu}$, and $\mathrm{H}$ attachment inside the MS detector could give the 170 amu observed ion peak.

The DMF-DMA derivatization agent that will be employed for GC-MS analysis of a comet nucleus by the COSAC instrumentation, on board the Rosetta Lander, was tested on our samples. The species reported here do not contain $\mathrm{COOH}$ or $\mathrm{NH}_{2}$ groups. Therefore no reaction with DMF-DMA takes place. The results are similar to those obtained without derivatization. The molecules HMT, HMT- $\mathrm{CH}_{3}, \mathrm{HMT}-\mathrm{OH}$, and HMT- $\mathrm{CH}(\mathrm{OH})-\mathrm{CHO}$ were also detected, with the same retention times. No additional species were found, since the detection of other residue components by means of GC-MS, like carboxylic acids, amides (Agarwal et al. 1985; Brigss et al. 1992) and amino acids (Muñoz Caro et al. 2002), requires a more specific analysis procedure that involves derivatization.

\subsection{Results}

A number of new species based on HMT were detected in the residues, i.e. methyl-HMT $\left(\mathrm{C}_{6} \mathrm{H}_{11} \mathrm{~N}_{4}-\mathrm{CH}_{3}\right)$, hydroxy-HMT $\left(\mathrm{C}_{6} \mathrm{H}_{11} \mathrm{~N}_{4}-\mathrm{OH}\right)$, methanyl-HMT $\left(\mathrm{C}_{6} \mathrm{H}_{11} \mathrm{~N}_{4}-\mathrm{CH}_{2} \mathrm{OH}\right)$, aminaldehyd-HMT $\left(\mathrm{C}_{6} \mathrm{H}_{11} \mathrm{~N}_{4}-\mathrm{NH}-\mathrm{CHO}\right)$ and methanyl-aldehydHMT $\left(\mathrm{C}_{6} \mathrm{H}_{11} \mathrm{~N}_{4}-\mathrm{CHOH}-\mathrm{CHO}\right)$. The species assigned to a particular experiment, see Table 1, correspond to a single GC-MS

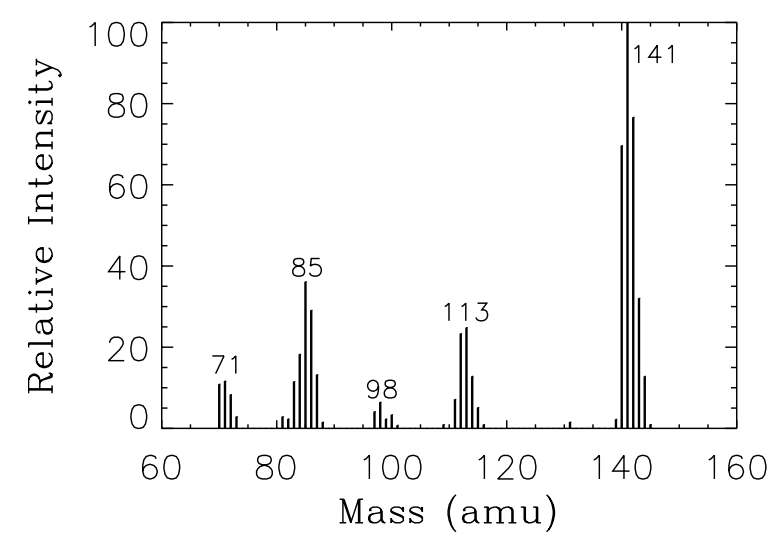

Fig. 7. Mass spectrum of HMT corresponding to the residue obtained from irradiation of the $\mathrm{H}_{2} \mathrm{O}: \mathrm{NH}_{3}: \mathrm{CH}_{3} \mathrm{OH}:{ }^{13} \mathrm{CO}:{ }^{13} \mathrm{CO}_{2}=2: 1: 1: 1: 1$ ice mixture.

run of the corresponding residue. The analysis of a different well on the same side of the Al-block sometimes gave a different number of detected species. Table 1 shows the results of the GC-MS runs with the largest number of products detected. The abundances of these products are also not very reproducible, which is may be not so surprising for trace abundance components. For two similar experiments, Exp. 4 and 5, the abundances of the products relative to HMT vary between 2.5, as observed for HMT-NH-CHO, and 10, for HMT- $\mathrm{CH}_{2} \mathrm{OH}$ (Table 1). This might be due to the fact that the residue is not homogeneously distributed throughout the surface. Instead, it forms droplets where it is more likely to have a concentration of the products above the detection limit, while the regions between droplets have very low concentrations. On the other hand, the solubility in methanol of the droplets was found to be lower than for the residue between droplets. Another possibility is that the relative abundances of the products present in droplets are truly different from those present in low density regions. Due to the uncertainty in the abundances, it was not possible to derive any trends with UV dose. However, all the products detected in our experiments were also found in the ${ }^{13} \mathrm{C}$ labelled sample. Only two molecules, HMT and HMT-OH, were detected in the ${ }^{15} \mathrm{~N}$-labelled experiment (Exp. 2, Table 1); both were labelled. The fact that all the species reported here were also present in the ${ }^{13} \mathrm{C}$-labelled experiment with the expected mass shifts, and that absolutely no species were found in the sides of the block were no deposition/irradiation took place excludes the possibility of contamination in our experiments. Therefore the HMT-based species are true products of photo- and thermal ice processing.

A small amount of HMT, about $1 \%$ of the residue, is produced by photolysis of CO: $\mathrm{H}_{2} \mathrm{O}: \mathrm{NH}_{3}$ ice mixtures (Briggs et al. 1992; Muñoz Caro \& Schutte 2003). Inclusion of $\mathrm{CH}_{3} \mathrm{OH}$ ice significantly enhances the HMT yield (Bernstein et al. 1995; Muñoz Caro \& Schutte 2003). In order to determine the contribution of $\mathrm{CO}$ and $\mathrm{CO}_{2}$, relative to $\mathrm{CH}_{3} \mathrm{OH}$, on the formation of HMT, the standard ice mixture with ${ }^{13} \mathrm{C}$-labelled $\mathrm{CO}$ and $\mathrm{CO}_{2}$, and unlabelled $\mathrm{CH}_{3} \mathrm{OH}$, was irradiated (Exp. 3). The mass spectrum obtained for HMT (Fig. 7) shows a molecular ion peak at masses ranging from 139 to $145 \mathrm{amu}$ due to inclusion of ${ }^{13} \mathrm{C}$ in the HMT molecule. From the peak heights of 
these ion masses it is possible to deduce the relative amount of carbon atoms, $R$, in the HMT originating from $\mathrm{CO}$ and $\mathrm{CO}_{2}$, as compared to the total amount of $\mathrm{C}$ atoms in HMT, i.e.

$R=\frac{N_{\mathrm{HMT}}^{\left(\mathrm{(TO}^{13} \mathrm{CO} \mathrm{CO}_{2}\right)}}{N_{\mathrm{HMT}}^{\left(\mathrm{CH} \mathrm{CH}_{3}{ }^{13} \mathrm{CO}+{ }^{13} \mathrm{CO}_{2}\right)}}=\frac{\sum_{m=141}^{146}(m-140) h_{m}}{\sum_{m=140}^{146} 6 h_{m}}=0.23$

where $h_{m}$ is the peak height for mass fragment $m$. The repetition of this experiment gave $R=0.28$, meaning that the result is rather reproducible. The same ratio was calculated for HMT-OH, giving $R=0.19$.

\subsection{Search for polycyclic aromatic hydrocarbons} (PAHs)

A search for polycyclic aromatic hydrocarbons (PAHs) was performed using $n$-hexane as the solvent. The instrument was calibrated using standards of simple $\mathrm{PAHs}$ such as phenanthrene $\left(\mathrm{C}_{14} \mathrm{H}_{10}, 3\right.$ rings $)$ and pyrene $\left(\mathrm{C}_{16} \mathrm{H}_{10}, 4\right.$ rings $)$. The standards of both compounds show clear signals in the chromatogram. Coronene $\left(\mathrm{C}_{24} \mathrm{H}_{12}, 7\right.$ rings) was not detected since the oven temperature $\left(\max .170^{\circ} \mathrm{C}\right)$ was not sufficiently high. A $n$-hexane solution of the standard residue was analyzed, showing a flat chromatogram, indicating the absence of PAHs in the residue material. Only when the residues are exposed to UV irradiation for a long period of time, such as the samples sent to space on the EURECA space platform, are PAHs able to form (Greenberg et al. 2000).

\subsection{Discussion}

The HMT-based species reported here are the residue components with the highest molecular mass yet found. To the best of our knowledge, this is the first synthesis of these species. The substitution of a $\mathrm{H}$ atom in HMT by a methyl-phenylboranediol radical was reported (Ivanova et al. 1975). Our results confirm that side groups can become attached to the HMT cage. The pathway of formation of HMT, see Fig. 8, as determined by the synthesis of this molecule from $\mathrm{H}_{2} \mathrm{CO}$ and $\mathrm{NH}_{3}$ in aqueous solution (Smolin \& Rapoport 1959; Walker 1964) might also be valid for ice irradiation experiments (Bernstein et al. 1995). Given the fact that HMT does not form till room temperature (Muñoz Caro \& Schutte 2003), HMT-based species are probably due to functional groups that were incorporated on the precursors of HMT at lower temperatures by substitution of a $\mathrm{H}$ atom. For instance, one of the $\mathrm{C}-\mathrm{H}$ bonds in hexahydro-1,3,5-triazine is broken by photodissociation and the radical site that results after loss of a $\mathrm{H}$ atom bonds to a functional group, e.g. a methyl group, forming 2-methyl-hexahydro-1,3,5-triazine, which after reaction with formaldehyde and ammonia (similar to steps 4 and 5, see Fig. 8) would result in methyl-HMT. The GC-MS detection of 2-methylamino-hexahydro-1,3,5-triazine and 2-diaminohexahydro-1,3,5-triazine in our residues (Meierhenrich et al. 2003) suggests this formation pathway is possible, but it needs to be confirmed.

Methanol, $\mathrm{CH}_{3} \mathrm{OH}$, is a better precursor of HMT than $\mathrm{CO}$ in ice photoprocessing experiments (Bernstein et al. 1995;
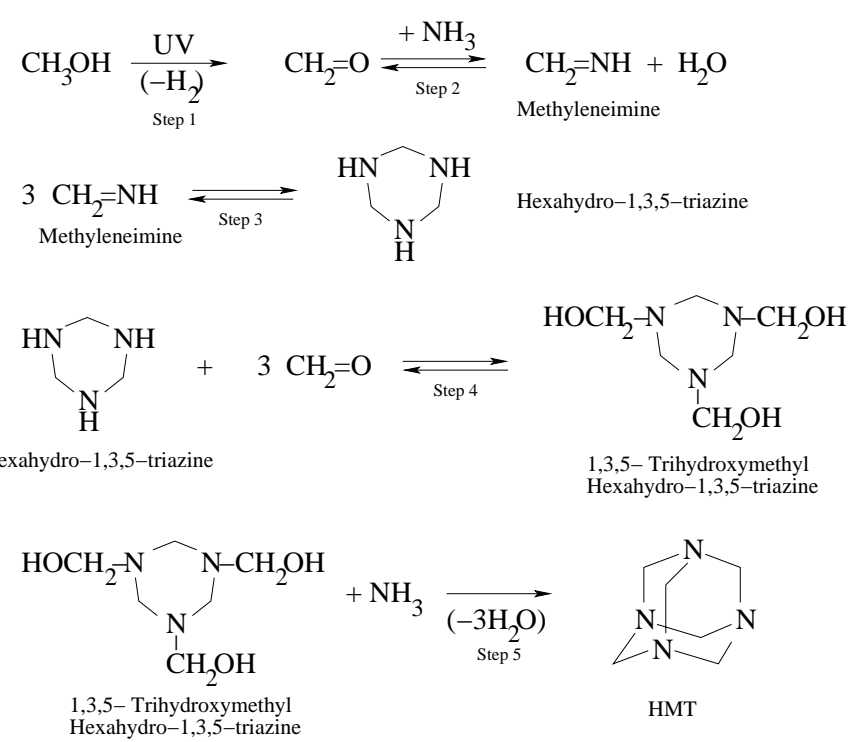

Fig. 8. Chemical pathway for the formation of HMT, after Smolin \& Rapoport (1959), Walker (1964) and Bernstein et al. (1995).

Muñoz Caro \& Schutte 2003). For the standard ice mixture it is found that $\sim 70 \%$ of the $\mathrm{C}$ atoms in HMT originates from $\mathrm{CH}_{3} \mathrm{OH}$, while the remaining $30 \%$ comes from $\mathrm{CO}$ and $\mathrm{CO}_{2}$ (Sect. 3.3, Exp. 3). This is most likely associated with the efficient formation of $\mathrm{H}_{2} \mathrm{CO}$ from $\mathrm{CH}_{3} \mathrm{OH}$ photolysis, as compared to $\mathrm{H}_{2} \mathrm{CO}$ production from irradiation of $\mathrm{H}_{2} \mathrm{O}$ and $\mathrm{CO}$ ice.

HMT was also produced by proton irradiation of $\mathrm{H}_{2} \mathrm{O}: \mathrm{CH}_{3} \mathrm{OH}: \mathrm{CO}: \mathrm{NH}_{3}=10: 5: 1: 1$ ice (Cottin et al. 2001). The search for HMT-based molecules, such as methyl-HMT and hydroxy-HMT, in these experiments give negative results (Cottin et al. 2001).

\section{Astrophysical implications}

The molecules reported in this paper would be very difficult to observe by astronomical infrared observations, since the broad features of the volatile ices $\left(\mathrm{H}_{2} \mathrm{O}, \mathrm{CH}_{3} \mathrm{OH}\right.$, etc.) hide those of minor organic components. Methyleneimine, a precursor of HMT (see Fig. 8), was detected in the mm region towards giant molecular clouds (Dickens et al. 1997). HMT could be searched for in the radio and $\mathrm{mm}$ wavelengths, in environments where ice mantles evaporate, like hot cores, although such large molecules will generally show weak lines. The HMT-based species, with expected abundances of the order of $1 \%$ of HMT or less (Table 1), would be more difficult to observe, although the larger dipole moment of these species, relative to HMT, will result in stronger rotational lines.

Comets preserve the most pristine material in the solar system. These bodies are thought to be formed by agglomeration of dust particles of interstellar and/or solar nebula origin in the outer parts of the solar nebula. Cometary dust is rich in organics, as much as $50 \%$ by mass (Fomenkova 1999). The aim of the COSAC instrumentation on board Rosetta is to perform in situ GC-MS analysis of a comet nucleus (Rosenbauer et al. 2003). The in situ analysis of cometary matter is probably the most reliable way to detect the organic products that originate from energetic processing of 
interstellar/solar nebula ice. The residue species which are detected by straightforward GC-MS analysis not involving derivatization, i.e. HMT and HMT-based molecules, could be good targets for the COSAC-Rosetta mission. Although due to poor reproducibility, no clear trend was measured in the abundance of HMT-based species relative to HMT in our experiments, it seems probable that their ratio is an indicator of the degree of processing of the pre-cometary ices. This is born out by the fact that the precursors of the HMT-based species should be formed after photoprocessing of the more basic precursors of pure HMT (Sect. 3.5). Therefore, the formation of HMT-based species requires a higher degree of photoprocessing than the formation of HMT. If found in comets, the presence of HMT-based species may therefore be an indicator of the amount of processing to which the ices were submitted before they were incorporated into comets. This could provide information on the physico-chemical conditions in the solar nebula.

Acknowledgements. We thank G. Lodder, J. Lugtenburg, G. Marel, H. S. Overkleeft, E. Pantoja and A. van der Gen for discussions on the chemical aspects. U.J.M. and G.M.M.C. thank the Max-PlanckInstitut für Aeronomie for fellowships. U.J.M. is grateful for a habilitation grant by the Deutsche Forschungsgemeinschaft.

\section{References}

Agarwal, V. K., Schutte, W. A., Greenberg, J. M., et al. 1985, Origins of Life and Evolution of the Biosphere, 16, 21

Allamandola, L. J., Sandford, S. A., \& Valero, G. J. 1988, Icarus, 76, 225

Bernstein, M. P., Sandford, S. A., Allamandola, L. J., Chang, S., \& Scharberg, M. A. 1995, ApJ, 454, 327

Bernstein, M. P., Dworkin, J. P., Sandford, S. A., Cooper, G. W., \& Allamandola, L. J. 2002, Nature, 416, 401

Briggs, R., Ertem, G., Ferris, J. P., et al. 1992, Origins of Life and Evolution of the Biosphere, 22, 287

Cottin, H., Szopa, C., \& Moore, M. H. 2001, ApJ, 561, L139

Dartois, E., Demyk, K., d'Hendecourt, L., \& Ehrenfreund, P. 1999, A\&A, 351, 1066

d'Hendecourt, L. B., Allamandola, L. J., Baas, F., \& Greenberg, J. M. 1982, A\&A, 109, L12

d'Hendecourt, L. B., Allamandola, L. J., Grim, R. J. A., \& Greenberg, J. M. 1986, A\&A, 158, 119

Dickens, J. E., Irvine, W. M., De Vries, C. H., \& Ohishi, M. 1997, ApJ, 479, 307

Ehrenfreund, P., Kerkhof, O., Schutte, W. A., et al. 1999, A\&A, 350, 240
Fomenkova, M. N., Chang, S., \& Mukhin, L. M. 1994, Geochim. Cosmochim. Acta, 58, 4503

Fomenkova, M. N. 1999, Space Sci. Rev., 90, 109

Gerakines, P. A., Schutte, W. A., Greenberg, J. M., \& van Dishoeck, E. F. 1995 , A\&A, 296, 810

Gerakines, P. A., Whittet, D. C. B., Ehrenfreund, P., et al. 1999, ApJ, 522,357

Gibb, E. L., Whittet, D. C. B., Schutte, W. A., et al. 2000, ApJ, 536, 347

Gibb, E. L., Whittet, D. C. B., \& Chiar, J. E. 2001, ApJ, 558, 702

Greenberg, J. M., \& Yencha, A. J. 1973, in Interstellar Dust and Related Topics, ed. J. M. Greenberg \& H. C. van de Hulst (Dordrecht: Reidel), 369

Greenberg, J. M. 1986, in The Galaxy and the Solar System (Tucson: Univ. of Arizona Press), 103

Greenberg, J. M., Gillette, J. S., Muñoz Caro, G. M., et al. 2000, ApJ, 531, L71

Hagen, W., Allamandola, L. J., \& Greenberg, J. M. 1979, Ap\&SS, 65, 215

Ivanova, E. A., Panchenko, S. I., Kolodkina, I. I., \& Yurkevich, A. M. 1975, J. Gen. Chem. USSR 1975, 773

Jenniskens, P., Baratta, G. A., Kouchi, A., et al. 1993, A\&A, 273, 583

Kissel, J., \& Krueger, F. R. 1987, Nature, 326, 755

Meierhenrich, U. J., Thiemann, W. H.-P., \& Rosenbauer, H. 2001, J. of Analytical and Applied Pyrolysis, 60, 13

Meierhenrich, U. J., Muñoz Caro, G. M., Schutte, W. A., et al. 2003, in preparation

Mennella, V., Muñoz Caro, G. M., Ruiterkamp, R., et al. 2001, A\&A, 367,355

Muñoz Caro, G. M., Ruiterkamp, R., Schutte, W. A., Greenberg, J. M., \& Mennella, V. 2001, A\&A, 367, 347

Muñoz Caro, G. M., Meierhenrich, U. J., Schutte, W. A., et al. 2002, Nature, 416, 403

Muñoz Caro, G. M., \& Schutte, W. A. 2003, A\&A, 412, 121

Oró, J. 1961, Nature, 190, 389

Rosenbauer, H., Goesmann, F., Roll, R., et al. 2003, ESA SP-1165 (Noordwijk: ESA), in press

Schutte, W. A., \& Khanna, R. K. 2003, A\&A, 398, 1049

Smolin, E. M., \& Rapoport, L. 1959, The chemistry of heterocyclic compounds. S. Triazines and derivatives (New York: Interscience), 26

Taban, I. M., Schutte, W. A., Pontoppidan, K. M., \& van Dishoeck, E. F. 2003, A\&A, 399, 169

Walker, J. F. 1964, Formaldehyde (New York: Reinhold Publishing Co.), 511

Weber, P., \& Greenberg, J. M. 1985, Nature, 316, 403 